\title{
Pseudotumor fibroso testicular: Aportación de un caso
}

\author{
Sánchez Bernal C, Muñoz Arias G*, Jiménez Romero ME, Navas Martínez C, Rodríguez-Rubio FI. \\ Servicio de Urología. *Servicio de Anatomía Patológica. Hospital Universitario de Puerto Real. Cádiz.
}

Actas Urol Esp. 2008;32(5):556-558

\section{RESUMEN}

PSEUDOTUMOR FIBROSO TESTICULAR: APORTACIÓN DE UN CASO

Aportamos un caso de pseudotumor fibroso del epidídimo como una localización aún más rara de esta proliferación difusa, nodular y osificada que puede ser difícil de diferenciar de los tumores sólidos. También hemos revisado las publicaciones hasta la fecha así como los posibles diagnósticos diferenciales.

Palabras clave: Epidídimo. Neoplasia testicular. Pseudotumor fibroso.

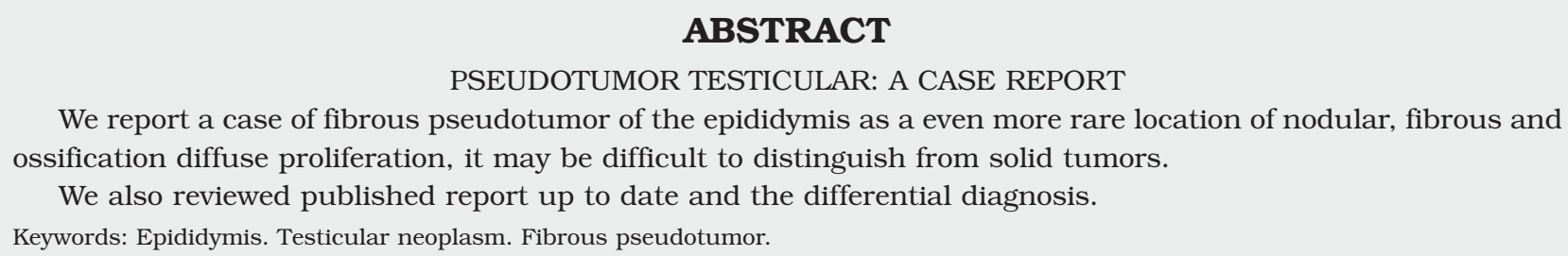

We report a case of fibrous pseudotumor of the epididymis as a even more rare location of nodular, fibrous and ossification diffuse proliferation, it may be difficult to distinguish from solid tumors.

We also reviewed published report up to date and the differential diagnosis.

Keywords: Epididymis. Testicular neoplasm. Fibrous pseudotumor.

$\mathrm{L}^{\mathrm{s}}$ os tumores sólidos paratesticulares son raras masas intraescrotales que se originan a partir de estructuras fasciales que tienen tejido conectivo: epidídimo, vaginal y cordón espermático, así como en tejidos escrotales: adiposo, fibroconectivo y muscular $^{1}$. Las túnicas testiculares son serosas derivadas del mesodermo con una superficie mesotelial y un conectivo subseroso que se asimila a la pleura y al peritoneo.

\section{CASO CLÍNICO}

Varón de 42 años con tumoración testicular izquierda de un año de evolución, descubierta accidentalmente sin antecedentes traumáticos o infectivos. A la exploración, las bolsas son simétricas, y en la izquierda se palpa una masa dura, abollonada, de consistencia pétrea y no dolorosa que sustituye al epidídimo y tercio superior del testículo. El resto del teste y del cordón son normales.

Los marcadores testiculares son normales y ecográficamente se trata de una masa heterogénea hipo e hiperecoica con áreas ecográficamente compatibles con litiasis (Fig. 1).

Explorado quirúrgicamente se le practicó orquiectomía inguinal izquierda. El informe anatomopatoló- gico refiere teste de $6 \times 3 \times 2,5 \mathrm{~cm}$ con nodulaciones osificadas en polo superior del testículo de $4,5 \mathrm{~cm}$ de longitud que se extienden por la túnica vaginal sin afectar al parénquima testicular. Existe proliferación fibrosa nodular e hialinizante que afecta a ambas vaginales. Los nódulos en su zona central se calcifican e incluso osifican confiriéndole un carácter pétreo (Figs. 2, 3 y 4): Periorquitis fibromatosa.

\section{COMENTARIO}

En general las masas paratesticulares son formaciones benignas en más del 90\% de los casos, al contrario de lo que sucede con las sólidas intratesticulares $^{2}$.

Por orden de frecuencia las masas sólidas extratesticulares más frecuentes son los tumores adenomatoides que asientan generalmente en epidídimo. Los pseudotumores fibrosos son la segunda causa de masa benigna paratesticular ${ }^{3}$ y aunque de etiología incierta se le atribuye una naturaleza reactiva y no neoplásica.

Estas masas fibromatosas han recibido múltiples denominaciones en la literatura médica, y aunque no consensuada hay cierta tendencia a englobarlas bajo la denominación genérica de Prolife- 


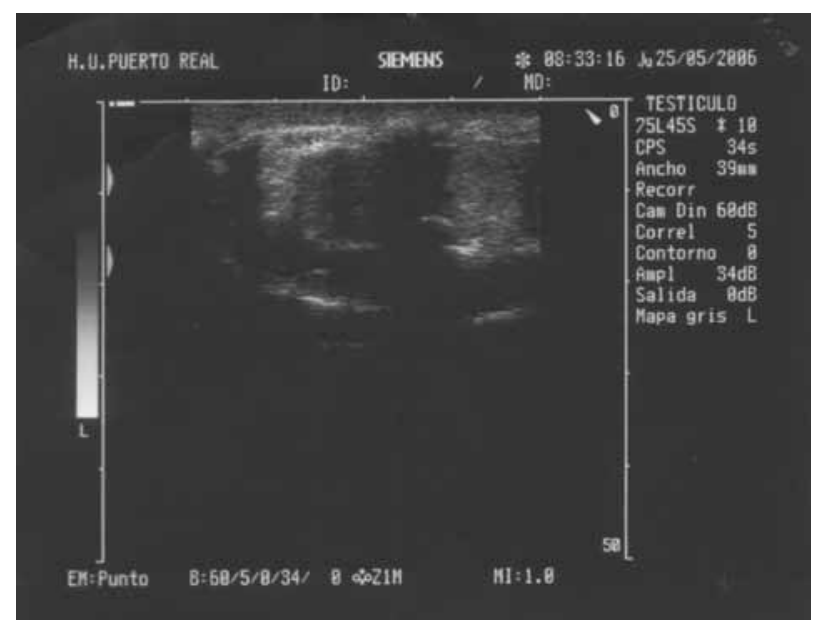

FIGURA 1. Ecografia. Imagen ecográfica de estructura heterogénea con áreas hipo e hiperecóicas y desestructuración epididimaria.

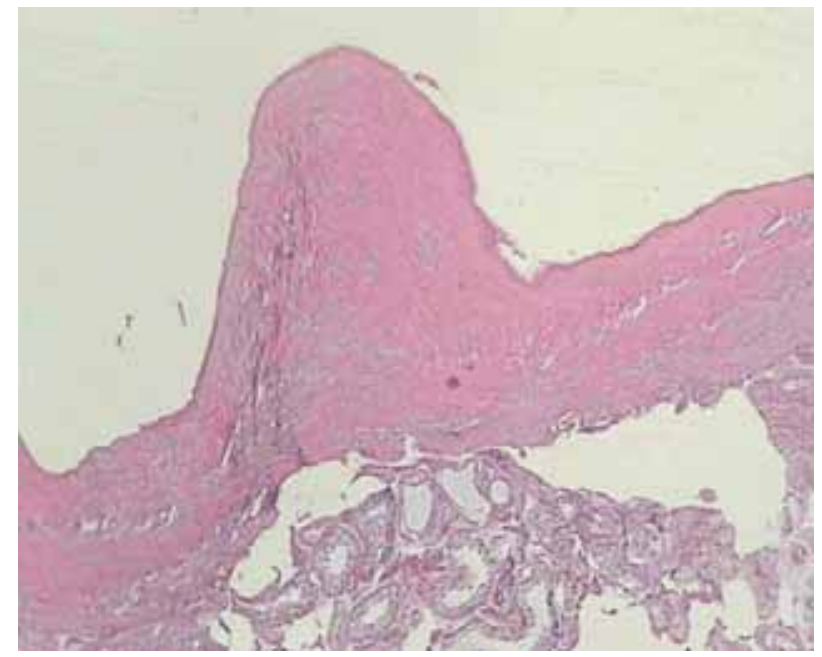

FIGURA 2. Anatomía patológica. Nódulo fibroso colágeno en vaginal adyacente a parénquima testicular normal.

ración Nodular Difusa Fibrosa ${ }^{4}$ sustituyendo la realizada por Goodwin y Vermooten en 1946 y que las agrupaba bajo la denominación de periorquitis crónica proliferativa ${ }^{5}$.

De entre las denominaciones que ha recibido dicha entidad destacan: periorquitis fibromatosa y pseudofibromatosa. periorquitis nodular, periorquitis crónica proliferativa o reactiva, fibrosis peritesticular y paratesticular inespecífica, pseudotumor fibroso, pseudotumor calcificante fibroso, tumor fibromatoso benigno paratesticular, proliferación difusa fibrosa paratesticular, proliferación nodular fibrosa paratesticular.
Todo ello hace más difícil el hallazgo exacto de su verdadera incidencia,aunque en nuestra revisión en Medline (fibrous pseudotumor,epididymis), aparecen publicados hasta la fecha 78 casos que se correspondan histopatológicamente a esta lesión, siendo el último caso el publicado por White et al en el $2006^{6}$.

Macroscópicamente se trata de tumores nodulares bien delimitados, difusos, únicos o múltiples, que sólo en el 10\% de los casos se localizan sobre epidídimo y cordón espermático 3. En ocasiones son masas móviles (ratón escrotal), y en general se asocian a fibrosis de las túnicas testiculares vaginal ó albugínea $^{7}$, y raramente encastran al testículo (periorquitis fibromatosa).

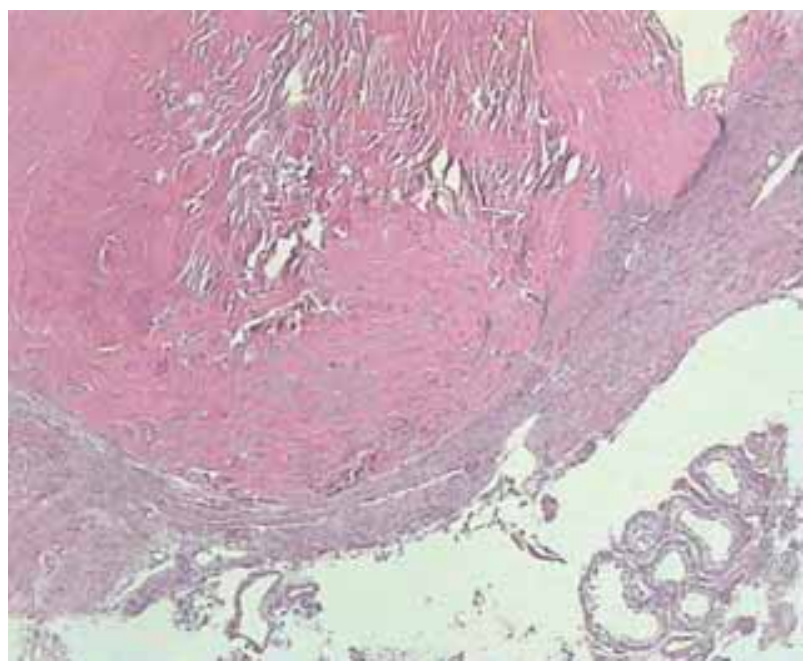

FIGURA 3. Nódulo fibroso colágeno en vaginal rodeado de proliferación fibrovascular.

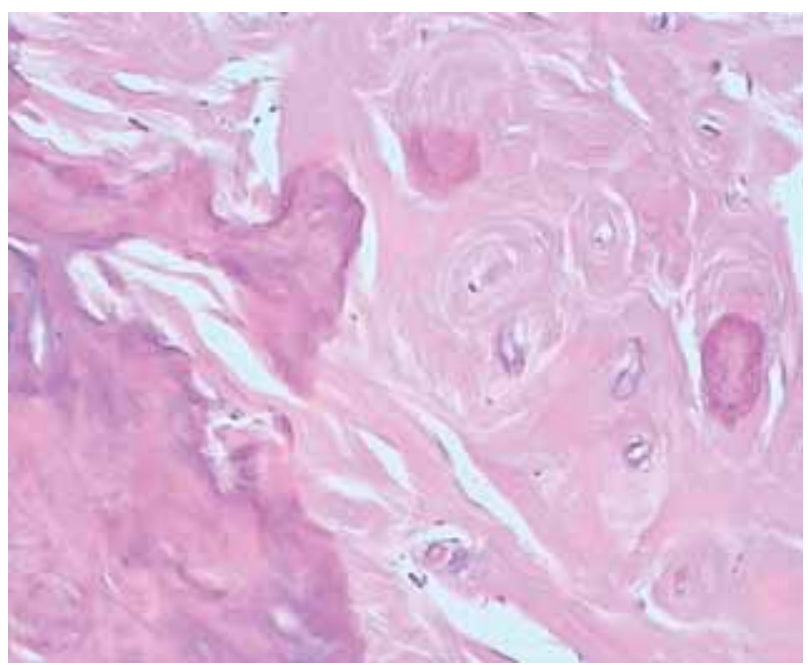

FIGURA 4. Calcificaciones y metaplasias óseas en nódulo fibroso hialino. 
Histológicamente, los nódulos se forman de tejido hialinizado y fibroblasto con estroma rícamente vascularizado con haces de colágena y focos de calcificación, y excepcionalmente osificado (como sucede en nuestro caso), y que puede en ocasiones precisar de diferencias con el mesotelioma o teratoma rico en elementos condroóseos, neurofibroma y leiomioma.

Recientemente, Jones ${ }^{8}$ ha agrupado estas lesiones en cuatro categorías a partir de los hallazgos anatomopatológicos:

- Angiomiofibroblastomas.

- Fibromas de túnicas testiculares.

- Fibromas de origen estromal.

- Pseudotumor fibroso.

La etiopatogenia de la lesión permanece desconocida. Mostofi la asocia a traumatismos o hidroceles atribuyéndoles una naturaleza reactiva, pero son muchos los casos publicados sin estos antecedentes $^{3}$. Su arco etário es amplio, aunque más frecuente entre la $3^{\mathrm{a}}$ y la $4^{\mathrm{a}}$ décadas de la vida. Existen casos infantiles hallados bajo la denominación de torsión testicular ${ }^{4}$.

La localización más frecuente es en la cubierta escrotal, (60\%), y el resto en epidídimo y cordón espermático ${ }^{9}$.

La ecografía no aporta datos definitivos, pudiendo ser lesiones hipo e hiperecoicas dependiendo de la densidad del colágeno, fibroblastos o calcificaciones con baja señal de intensidad en relación al testículo en señales de resonancia nuclear magnética $^{10}$.

Cuando existe sospecha de pseudotumor fibroso testicular hay que realizar una biopsia extemporánea y cirugía conservadora. El tratamiento depende del tipo de lesión, encontrándose casos aislados de exéresis de lesiones localizadas, como nódulos separados del testículo, pero en general el tratamiento fue la orquiectomía. Vates et al. consideran que el límite de la resección es de $3 \mathrm{~cm}$ de diámetro pero si el crecimiento es paratesticular, tumores más grandes pueden extirparse intentando preservar el testículo, como el caso publicado por White et $\mathrm{al}^{6}$.

En nuestro caso no pudo llevarse a cabo cirugía conservadora por alta sospecha de malignidad y por imposibilidades técnicas, dado el volumen tumoral.

\section{REFERENCIAS}

1. Woodward PJ, Schwab CM, Sesterhenn IA. Extratesticular scrotal masses. Radiologic pathologic correlation. Radiographics. 2003;23(1): 215-240.

2. Tobias-machado M, Corrêa Lopes Neto A, Heloisa Simardi L, Borrelli M, Wroclawski ER. Fibrous Pseudotumor of tunica vaginalis and epididymis. Urology. 2000;56(4):669-672.

3. Mostofi FK, Price EB. Tumor of the male genital system. Atlas of tumor pathology, $2^{\text {nd }}$ series. Fascimile 8. Armed Forced Institue of Pathology, 1973; 151-154.

4. Ruiz Liso J.M, Ruiz García J, Aguas Arias C, Vaillo Vinagre A, Martin Gutierrez A et al. Proliferación nodular y difusa fibrosa de la túnica vaginal del testículo. Rev. Esp. Patol 2004; 37(1): 91-98.

5. Goodwin WE, Vermooten V. Multiple Fibromata of tunica vaginalis testis of proliferative type of chronic periorquitis. J. Urol. 1946;56:430.

6. White WM, Hilsenbeck, Waters WB. Fibromatous periorchitis of testis. Urology 2006 Mar;67 (3): 623.e 15-6.

7. Gögüs O, Bulay O, Yurdakul T, Bedük Y. A rare scrotal mass: fibrous pseudotumor of epididymis. Urol. Int. 1990;45(1):6364.

8. Jones MA, Young RH, Scully RE. Bening fibromatous tumors of the testis and paratesticular region:A report of 9 cases with a proposed classification of fibromatous tumours and tumor-like lesions. Am. Surg. Pathol. 1997;21(3):296-305.

9. Srigley J R, Hartwick R W J.Tumors and cyst of the paratesticular region. Pathol Ann 1990, 25 (PT2):51-108.

10. al-Otaibi L, Whitman GJ, Chew FS. Fibrous psudotumor of the epididymis. AJR Am J Roentgenol. 1997;168(6):1586-1587.

Correspondencia autor: Dr. C. Sánchez Bernal Servicio de Urología. Hospital Univ. de Puerto Real Ctra. Nac. IV, Km. 665 - 11510 Puerto Real (Cádiz) Tel: 956005000

E-mail autor: csbernal@hotmail.com

Información artículo: Notas clínicas

Trabajo recibido: noviembre 2006

Trabajo aceptado: diciembre 2007 